\title{
Contenidos teóricos de las materias generales y especializadas en los planes de estudios de las diplomaturas de ciencias de la salud
}

\author{
Theoretic contents of general and specialized subjects in core curricula \\ of health sciences professions
}

José Antonio Arias Navalón ${ }^{\mathrm{a}}$ y $\mathrm{M}^{\mathrm{a}}$ Luz Cuadrado Pérez ${ }^{\mathrm{b}}$

${ }^{a}$ Facultad de Ciencias de la Salud. Universidad Alfonso X el Sabio.Villanueva de la Cañada. Madrid.

${ }^{b}$ Facultad de Ciencias de la Salud. Universidad Rey Juan Carlos. Alcorcón. Madrid.

Objetivo: Evaluar cuantitativamente los contenidos teóricos, generales y especializados, de los planes de estudios de las diplomaturas de ciencias de la salud en España. A partir de esos datos se harán algunas recomendaciones y se destacarán aspectos que podrían necesitar modificaciones.

Diseño: Revisión sistemática.

Emplazamiento y material de estudio: Planes de estudios de las diplomaturas de ciencias de la salud en España: Enfermería, Fisioterapia, Logopedia, Nutrición humana y dietética, Óptica y optometría, Podología y Terapia ocupacional. Mediciones: Número de horas teóricas dedicadas a materias troncales, detallando su carácter general o especializado.

Resultados y conclusiones: En conjunto, los contenidos especializados y generales suponen, respectivamente, el 66,7 y el $33,3 \%$. La mayoría de las carreras tienen más horas asignadas a materias especializadas. Los resultados oscilan entre la ausencia de materias troncales generales en las carreras de Óptica y optometría y de Logopedia y el $71,4 \%$ de carga lectiva de carácter general en la carrera de Terapia ocupacional. La carencia de conocimientos generales sobre la salud y la enfermedad puede tener consecuencias negativas en la práctica diaria y en las expectativas que tienen para hacer investigación los profesionales implicados.

Palabras clave: Diplomaturas de ciencias de la salud.

Plan de estudios. Materias troncales.

Correspondencia:

José Antonio Arias Navalón

Despacho D-335

${ }^{\mathrm{a}}$ Facultad de Ciencias de la Salud

Universidad Alfonso X el Sabio

Avda de la Universidad, 1

28691 Villanueva de la Cañada, MADRID

E-mail: josari@uax.es

${ }^{\mathrm{b}}$ Facultad de Ciencias de la Salud

Universidad Rey Juan Carlos

Avda Atenas s/n

28922 Alcorcón, Madrid
Objective: To assess general and specialized theoretic contents of core curricula of health professions in Spain, in order to make some recommendations to improve these curricula and to highlight some areas needing further modifications.

Design: Systematic revision.

Setting and study selection: Core curricula of health professions in Spain: Nursing, Physical therapy, Speech-language pathology, Nutrition and dietetics, Optometry, Podiatry and Occupational therapy.

Measurements: Number of theoretic hours devoted to both general and specialized subjects.

Results and conclusions: Overall, specialized and general contents are $66.7 \%$ and $33.3 \%$, respectively. Most careers have more hours focused on specialized subjects, with results ranging between the absence of general subjects in Optometry and Speech-language pathology careers and $71.4 \%$ of general contents in Occupational therapy. The lack of general health and disease knowledge might have important implications for daily practice and research expectatives of these health professions.

Key words: Health sciences professions. Core curriculum. Core subjects.

INTRODUCCIÓN

En el sistema universitario español, son materias troncales de una carrera aquellas que obligatoriamente deben incluirse en todos los planes de estudios que conduzcan a un mismo título oficial. Estas materias, con la duración mínima en horas teóricas y prácticas, están recogidas en las directrices generales propias de cada titulación. Se trata, en otras palabras, de contenidos docentes que el legislador 
Tabla 1. Número de créditos teóricos por carrera y tipo de materia.

\begin{tabular}{|c|c|c|c|}
\hline Carrera & Créditos generales & Créditos especializados & Créditos totales \\
\hline ENF & $16(27)$ & $44(73)$ & 60 \\
\hline FTP & $37(59)$ & $26(41)$ & 63 \\
\hline LOG & $0(0)$ & $40(100)$ & 40 \\
\hline NHD & $21(28)$ & $53,5(72)$ & 74,5 \\
\hline OPT & $0(0)$ & $78(100)$ & 78 \\
\hline POD & $29(39)$ & $45(61)$ & 74 \\
\hline TOC & $50(71)$ & $20(29)$ & 70 \\
\hline Total: & $153(33)$ & $306,5(67)$ & 459,5 \\
\hline
\end{tabular}

Entre paréntesis se muestran los porcentajes respecto al total de créditos teóricos de la carrera. ENF: Enfermería; FTP: Fisioterapia; LOG: Logopedia; NHD: Nutrición humana y dietética; OPT: Óptica y optometría; POD: Podología; TOC: Terapia ocupacional.

estima que son imprescindibles para la formación de los futuros titulados.

Todas las diplomaturas de ciencias de la salud tienen una duración de tres años académicos. Parece deseable que en ese tiempo se impartan conocimientos generales acerca del ser humano sano y enfermo, en sus aspectos físico, psíquico y social, antes de abordar los conocimientos específicos de la carrera. En este trabajo nos proponemos trazar un cuadro descriptivo del volumen de horas teóricas en materias troncales y su carácter general o especializado en las distintas diplomaturas de ciencias de la salud.

\section{MATERIAL Y MÉTODOS}

Se tomaron como fuente las directrices generales propias de las diplomaturas de la salud: Enfermería ${ }^{1}$ (ENF), Fisioterapia ${ }^{2}$ (FTP), Logopedia ${ }^{3}$ (LOG), Nutrición Humana y Dietética ${ }^{4}$ (NHD), Óptica y Optometría $^{5}$ (OPT), Podología ${ }^{6}$ (POD) y Terapia Ocupacional $^{7}$ (TOC). En el marco legislativo español la NHD y la OPT se clasifican como carreras experimentales, antes que sanitarias. No obstante, nosotros las incluimos en este trabajo porque dichas titulaciones inciden de manera obvia en temas de salud. Los dos autores estudiaron independientemente todas las materias con horas teóricas de cada una de las directrices y las clasificaron como generales 0 especializadas, atendiendo a los siguientes criterios:

1. Materias generales: aquellas que permiten adquirir un conocimiento global del cuerpo humano, sano o enfermo, sin ceñirse a un enfoque parcial o a un aspecto particular. Se admiten los enfoques físico, psíquico y social de la salud y la enfermedad. No mencionan aspectos específicos de la carrera en que se estudian, ni permitirían identificar ésta.

Ejemplos: Estructura y función del cuerpo humano (ENF, FTP, NHD, POD y TOC) y Fisiopatología (NHD).

2. Materias especializadas: aquellas que no cum- plen los requisitos anteriores. En muchos casos, permiten reconocer fácilmente la carrera en la cual se imparten. No serían pertinentes como materias troncales en otra titulación.

Ejemplos: Quiropodología (POD) y Teoría y técnicas de la terapia ocupacional (TOC).

Quedaron excluidas del análisis, por tener sólo créditos prácticos, las materias Estancias clínicas de FTP y Practicum de LOG.

A la hora de la clasificación, se tomaron como referencia principal los descriptores que figuran en las directrices de cada carrera, los cuales describen brevemente el contenido de cada materia. El programa que debe elaborar el profesor responsable de la asignatura debe respetar las líneas maestras definidas por dichos descriptores. Secundariamente, en el trabajo de clasificación se valoraron el nombre de la asignatura y las áreas de conocimiento vinculadas a ella, que quedan también reflejadas en las directrices mencionadas.

Los dos autores de este artículo distribuyeron de forma independiente las materias en alguna de las dos categorías indicadas. Posteriormente se analizó el acuerdo en los resultados mediante la prueba kappa $(k)$ de concordancia entre observadores. Después se llegó a una clasificación de consenso entre ambos, tras comprobar que la concordancia previa al consenso era aceptable. Finalmente, se obtuvo el número de créditos teóricos correspondientes a cada grupo de materias (generales o especializadas) en cada carrera. En todos los casos, un crédito teórico equivale a 10 horas lectivas.

\section{RESULTADOS}

El valor del índice kappa fue de 0,82 (intervalo de confianza del $95 \%$ : 0,67 a 0,97 ), lo que representa un acuerdo excelente entre observadores. El número total de créditos teóricos de materias generales y 
especializadas en las distintas carreras se muestra en la tabla 1. En conjunto, los créditos generales y especializados son, respectivamente, el $33,3 \%$ y el $66,7 \%$ de la carga lectiva total en horas teóricas. Al considerar las titulaciones por separado, el espectro se extiende entre dos situaciones extremas: la carencia absoluta de créditos teóricos generales en el caso de OPT y LOG, por un lado, y la abundancia de créditos generales de TOC, con un $71,4 \%$ de la carga total teórica, por otro. Sólo dos titulaciones TOC y FTP- tienen mayor cantidad de créditos teóricos generales que especializados. En la figura 1 se muestran gráficamente los créditos teóricos totales

Fig. 1. Créditos teóricos totales por carreras, en orden descendente de izquierda a derecha. Para abreviaturas, ver texto.

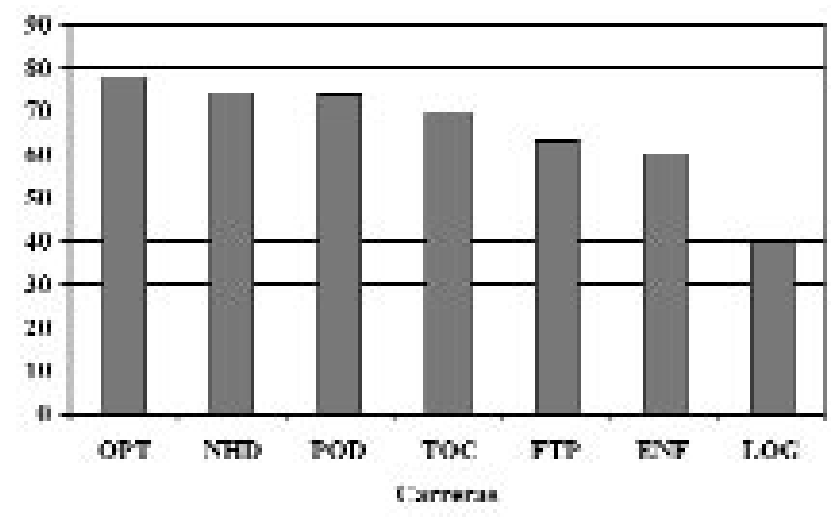

Fig. 2. Distribución de créditos teóricos generales y especializados por carreras.

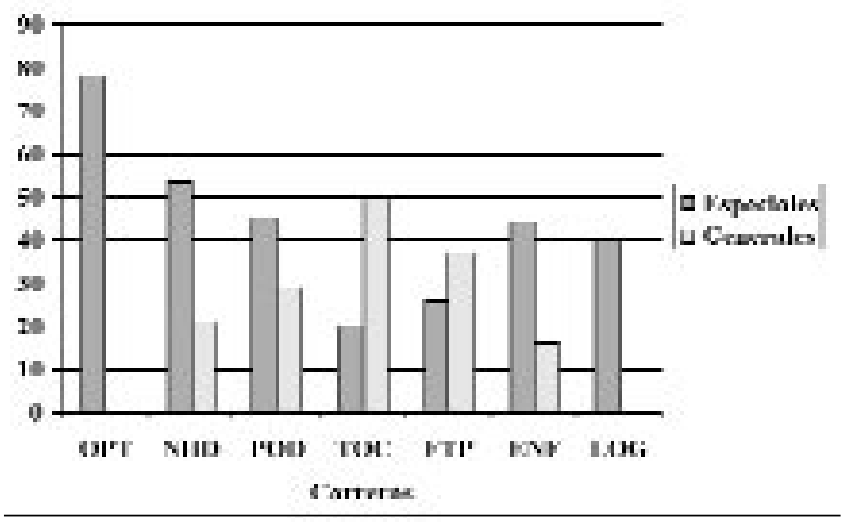

por carreras y en la figura 2 la distribución según el carácter general o especializado.

\section{DISCUSIÓN}

Resulta notoria la gran diferencia en la proporción de materias generales y especializadas entre titulaciones. Hay que advertir, ciertamente, que en la rea- lidad académica, las universidades añaden en sus planes de estudios materias obligatorias y optativas que completan e intensifican los contenidos generales y especializados y que pueden compensar, en parte, estas diferencias. No obstante, las asignaturas troncales suponen el marco legal mínimo y marcan un estilo en la formación del futuro titulado.

Existen, al menos, dos preguntas obvias en relación con estos datos: 1) ¿se ajustan estas proporciones a las competencias que la sociedad quiere del diplomado? y 2) ¿qué grado de autonomía y desarrollo profesional cabe esperar de un diplomado que carece de una formación general de la salud o el enfermar humano? Se trata de cuestiones siempre debatidas.

Algunos autores señalan que un aspecto negativo de la reforma universitaria de 1987 es la disminución de conocimientos básicos, con una mayor especialización y una formación parcelada, contrario todo ello a las corrientes internacionales de renovación académica de los estudios universitarios ${ }^{8}$. Es cierto que el estudio en profundidad de un determinado campo genera una sólida competencia en ese campo concreto, pero conduce fácilmente a una visión limitada y además debilita la capacidad de aprender cosas nuevas cuando la materia aprendida se ha quedado ya obsoleta 9 .

En el ámbito concreto de las ciencias de la salud existen razones adicionales, de máxima relevancia, que hacen fundamental que todos los profesionales adquieran conocimientos generales relativos al ser humano en condiciones normales y patológicas ${ }^{10}$. Estos conocimientos permitirán, por ejemplo, tratar el problema de salud que sufre el paciente en un contexto global. Además posibilitarán la identificación de datos que hagan aconsejable remitir al paciente a otro profesional. Finalmente, capacitarán al trabajador sanitario para actuar ante una emergencia que requiera primeros auxilios.

Se debe reconocer que no son iguales las competencias de las diversas profesiones y que las necesidades de formación general entre titulaciones pueden ser diferentes. La capacidad para desempeñar las tareas que son específicas de cada diplomatura con los planes de estudio vigentes no se pone en duda: el nivel de las disciplinas básicas es seguramente suficiente para la práctica diaria en todas estas profesiones. Sin embargo, los autores de este artículo manifiestan su preocupación por el contraste entre el grado creciente de autonomía que reclaman muchos profesionales para su actividad profesional e investigadora y el escaso bagaje general 
detectado en los contenidos mínimos legales requeridos en algunas de las titulaciones estudiadas. Estos hechos deberían ser tenidos en cuenta ante la proximidad de la nueva reforma académica.

\section{REFERENCIAS}

1. Real Decreto $1466 / 1990$, de 26 de octubre, por el que se establece el título universitario oficial de Diplomado en Enfermería y las directrices generales propias de los planes de estudios conducentes a la obtención de aquel (BOE, número 278, de 20-11-1990).

2. Real Decreto 1414/1990, de 26 de octubre, por el que se establece el título universitario oficial de Diplomado en Fisioterapia y las directrices generales propias de los planes de estudios conducentes a la obtención de aquel (BOE, número 278, de 20-11-1990).

3. Real Decreto 1419/1991, de 30 de agosto, por el que se establece el título universitario oficial de Diplomado en Logopedia y las directrices generales propias de los planes de estudios conducentes a la obtención de aquel (BOE, número 243, de 10-10-1991).

4. Real Decreto 433/1998, de 20 de Marzo, por el que se establece el título universitario oficial de Diplomado en Nutrición Humana y Dietética y las directrices generales propias de los planes de estudios conducentes a la obtención de aquel (BOE, número 90, de 15-04-1998).
5. Real Decreto 1419/1990, de 26 de octubre, por el que se establece el título universitario oficial de Diplomado en Optica y Optometría y la directrices generales propias de los planes de estudios conducentes a la obtención de aquel (BOE, número 278, de 20-11-1990).

6. Real Decreto 649/1988, de 24 de junio, por el que se transforman los estudios de Podología en primer ciclo universitario conducente al título de Diplomado universitario en Podología y se establecen las directrices generales propias de los correspondientes planes de estudio (BOE, número 153, de 27-06-1988).

7. Real Decreto $1420 / 1990$, de 26 de octubre, por el que se establece el título universitario oficial de Diplomado en Terapia Ocupacional y las directrices generales propias de los planes de estudios conducentes a la obtención de aquel (BOE, número 278, de 20-11-1990).

8. Michavila F, Calvo B. La universidad española hoy. Propuestas para una política universitaria. Madrid: Síntesis; 1998.

9. Husén T. Tradiciones, crisis y futuro de la universidad occidental. En: García Hoz V (dir.). La Educación Personalizada en la Universidad, vol. 27 del Tratado de Educación Personalizada. Madrid: Rialp; 1996. p. 56-80. 10. Wray D, Lowe GDO, Dagg JH, Felix DH, Scully C. Textbook of general and oral medicine. Edimburgo: Churchill Livingstone; 1999. 